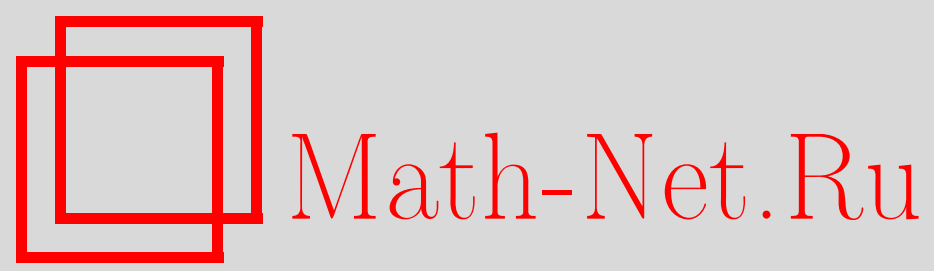

Е. В. Писковский, О классическом и функциональном подходах к механике, Вестн. Сам. гос. техн. ун-та. Сер. Физ.-мат. науки, 2011, выпуск 1(), 134139

DOI: https://doi.org/10.14498/vsgtu895

Использование Общероссийского математического портала Math-Net.Ru подразумевает, что вы прочитали и согласны с пользовательским соглашением

http: //www . mathnet.ru/rus/agreement

Параметры загрузки:

IP: 54.224 .187 .69

26 апреля 2023 г., 17:29:10 
УДК 517.958

\section{О КЛАССИЧЕСКОМ И ФУНКЦИОНАЛЬНОМ ПОДХОДАХ К МЕХАНИКЕ}

\section{E. В. Писковский}

Московский физико-технический институт (государственный университет), 141700, Московская обл., Долгопрудный, Институтский переулок, 9.

E-mail: piskovskiy@mi.ras.ru

Рассматривается вопрос о соответствии классической траектории ангармонического осииллятора и усредненной траектории в рамках функционалъного подхода. Оченивается величина характерного времени расхождения траекторий в зависимости от дисперсии начальных значений.

Ключевые слова: функциональная механика, классическая механика, модельная система, ангармонический осииллятор, пороговое время.

Основополагающим принципом в подходе Ньютона является редукционизм: чтобы понять любое сложное явление, необходимо разложить его на элементарные составляющие. Единственные характеристики, которые различают частицы друг от друга, - их координата в пространстве и скорость. Если известны начальные координаты и скорости частиц, составляющих систему, и все силы, действующие на эти частицы, тогда можно с наперед заданной точностью указать эволюцию системы. Эволюция системы при таком методе описания является обратимой по времени.

Однако ньютоновское описание мира не вполне удовлетворительно, по крайней мере, по двум причинам. Во-первых, в определении начального положения и начальной скорости присутствует неопределенность. Во-вторых, в рамках ньютоновского подхода нельзя разрешить проблему обратимости по времени (см. $[1,2])$; обсуждение проблемы необратимости можно найти, например, в [3]. В работах И. В. Воловича [1,2] был представлен новый подход к классической механике, в рамках которого основным понятием является не траектория, а функция распределения вероятности. В рамках рассматриваемого подхода (см. также [4-7]) изучаются средние траектории и поправки к решениям уравнений Ньютона.

В настоящей работе рассматривается соответствие между траекториями ангармонического осциллятора, полученными в рамках подхода Ньютона, и траекторией, усредненной согласно функционально-механическому подходу, с целью оценки зависимости характерного времени расхождения этих траекторий от величины дисперсии распределения координаты и импульса. Данное исследование вдохновлено работой Инуэ (Inoue), Ойяа (Ohya) и И. В. Воловича [8], в которой рассматривается соответствие классической и квантовой траекторий динамической системы, описываемой преобразованием пекаря (о преобразовании пекаря см., например, $[9,10]$ ).

Фундаментальным уравнением описания динамики на микроскопическом уровне предложенного функционально-механического подхода является урав-

Евгений Викторович Писковский, аспирант, факультет управления и прикладной математики. 
нение Лиувилля для функции распределения вероятности. Широко известно, что уравнение Лиувилля применяется в статистической механике для описания движения газа, однако отметим, что в данном подходе это уравнение будет использовано для описания одной частицы в пространстве.

Уравнение Лиувилля на многообразии $\Gamma$, на котором введены координаты $x=\left(x^{1}, x^{2}, \ldots, x^{k}\right)$ и векторное поле $v=v(x)=\left(v^{1}, v^{2}, \ldots, v^{k}\right)$, имеет вид

$$
\frac{\partial \rho}{\partial t}+\sum_{i=1}^{k} \frac{\partial}{\partial x^{i}}\left(\rho v^{i}\right)=0,
$$

где $\rho=\rho(x, t)$ - функция плотности вероятности. Решение задачи Коши для уравнения (1) с начальными данными $\left.\rho\right|_{t=0}=\rho_{0}(x)$ может быть записано в виде $\rho(x, t)=\rho_{0}\left(\varphi_{-t}(x)\right)$. Здесь $\varphi_{t}(x)$ - фазовый поток вдоль решения характеристического уравнения $\dot{x}=v(x)$.

Пусть $(q, p)$ - точка в $\mathbb{R}^{2}$ (фазовое пространство), $t \in \mathbb{R}$ - время. Состояние классической частицы в момент времени $t$ описывается функцией плотности вероятности $\rho=\rho(q, p, t)$ (здесь $\delta$-функция недопустима в качестве функции распределения).

Предполагается, что $\rho=\rho(q, p, t) \geqslant 0$ - непрерывно дифференцируемая и интегрируемая функция, $t \in \mathbb{R}$ и

$$
\int_{\mathbb{R}^{2}} \rho(q, p, t) d q d p=1
$$

Пусть $f=f(q, p)$ - функция на фазовом пространстве. Определим среднее значение функции $f$ в момент времени $t$ через интеграл:

$$
\langle f\rangle(t)=\int_{\mathbb{R}^{2}} f(q, p) \rho(q, p, t) d q d p .
$$

Движение точечной массы вдоль линии в потенциальном поле описывается уравнением

$$
\frac{\partial \rho}{\partial t}=-\frac{p}{m} \frac{\partial \rho}{\partial q}+\frac{\partial V(q)}{\partial q} \frac{\partial \rho}{\partial p}
$$

Здесь $V(q)$ - потенциальное поле, а $m>0$ - масса.

Рассмотрим задачу Коши для уравнения (2) с начальным условием $\left.\rho\right|_{t=0}=$ $=\rho_{0}(q, p)$. Средние траектории определяются следующим образом:

$$
\langle q\rangle(t)=\int_{\mathbb{R}^{2}} q \rho(q, p, t) d q d p=\int_{\mathbb{R}^{2}} q(t) \rho_{0}(q, p) d q d p,
$$

где $q(t)$ - классическая траектория точечного тела, отвечающая уравнению Ньютона.

Ангармонический осциллятор. Предлагается рассмотреть точечную массу, двигающуюся в поле, описываемом потенциалом

$$
V(q)=\frac{1}{2} \omega_{0}^{2} q^{2}+\frac{1}{4} \varepsilon q^{4},
$$


где $\omega_{0}>0, \varepsilon>0$-малый параметр. Координата $q(t)$ меняется во времени согласно уравнению Ньютона:

$$
\ddot{q}+\omega_{0}^{2} q=-\varepsilon q^{3}
$$

с начальными условиями

$$
\left.q\right|_{t=0}=b_{0},\left.\quad \dot{q}\right|_{t=0}=0 .
$$

Точное решение задачи Коши для уравнения (4) хорошо известно, но для простоты используется приближенное решение, полученное с помощью метода Крылова-Боголюбова (см., например, [11]):

$$
q_{\mathrm{KB}}\left(t, b_{0}\right)=a \cos (t \omega)+\varepsilon \frac{a^{3}}{32 \omega_{0}^{2}} \cos (3 t \omega)+O\left(\varepsilon^{2}\right),
$$

где $a$ - постоянная интегрирования, а частота $\omega=\omega(a)$ задаётся следующим соотношением:

$$
\omega=\omega(a)=\omega_{0}\left(1+\frac{3}{8} a^{2} \varepsilon+O\left(\varepsilon^{2}\right)\right) .
$$

Согласно начальному условию (5)

$$
a+\varepsilon \frac{a^{3}}{32 \omega_{0}^{2}}=b_{0} .
$$

Тогда для средней траектории имеем

$$
\langle q\rangle(t, \sigma)=\int_{\mathbb{R}} q_{\mathrm{KB}}(t, b) \rho_{0}(b) d b,
$$

где

$$
\rho_{0}(b)=\frac{1}{\sqrt{\sigma \pi}} \exp \left(-\frac{\left(b-b_{0}\right)^{2}}{\sigma}\right)
$$

- функция распределения, а $\sigma / 2>0$ - дисперсия параметра $b$.

Так как $\langle q\rangle(0, \sigma)=q_{\mathrm{KB}}\left(t, b_{0}\right)=b_{0}$, то при малых $t$ разница между функциями $\langle q\rangle(0, \sigma)$ и $q_{\mathrm{KB}}\left(t, b_{0}\right)$ мала. Необходимо определить время $t_{C}$ такое, чтобы модуль разности между двумя рассматриваемыми функциями был равен некоторому пороговому значению, и зависимость $t_{C}=t_{C}(\sigma)$. Эти вопросы уже рассматривались в [8] для квантовых систем.

Сравнение траекторий. В работе [2] доказано, что в любой момент времени $t$ верно

$$
\lim _{\sigma \rightarrow 0}\langle q\rangle(t, \sigma)=q_{\mathrm{KB}}\left(t, b_{0}\right),
$$

вследствие чего следует ожидать роста $t_{C}(\sigma)$ при $\sigma \rightarrow 0$.

Пусть $\omega_{0}=1$. Действительные корни (6) даются выражением [12]:

$$
a=a(b)=8 \sqrt{\frac{2}{3 \varepsilon}} \operatorname{sh}\left(\frac{1}{3} \operatorname{arcsh}\left(\frac{3}{8} \sqrt{\frac{3 \varepsilon}{2}} b\right)\right) .
$$


После замены переменной в (7) получим

$$
\begin{aligned}
\langle q\rangle(t, \sigma)=\frac{1}{\sqrt{\pi}} \int_{\mathbb{R}}(a(z \sqrt{\sigma} & \left.+b_{0}\right) \cos \left(t \omega\left(z \sqrt{\sigma}+b_{0}\right)\right)+ \\
& \left.+\frac{\varepsilon\left(a\left(z \sqrt{\sigma}+b_{0}\right)\right)^{3}}{32} \cos \left(3 t \omega\left(z \sqrt{\sigma}+b_{0}\right)\right)\right) e^{-z^{2}} d z .
\end{aligned}
$$

С помощью метода перевала можно получить грубую оценку асимптотической зависимости характерного времени $t_{C}$ от $\sigma$ при $\sigma \rightarrow 0: t_{C}=O(1 / \sqrt{\sigma})$ при $\sigma \rightarrow 0$.

Численный подход. Рассмотрим функцию $\Delta(t)=\left|\langle q\rangle(t, \sigma)-q_{\mathrm{KB}}\left(t, b_{0}\right)\right|$. Тогда $t_{C}=t_{C}(\sigma, C)$ есть такой момент времени, что $\left|\Delta\left(t_{C}\right)\right|=C$.

Пусть $C=\varepsilon q_{\mathrm{KB}}\left(0, b_{0}\right)$, тогда характерное время отвечает следующему уравнению:

$$
\left|\Delta\left(t_{C}\right)\right|=\varepsilon q_{\mathrm{KB}}\left(0, b_{0}\right),
$$

где $\varepsilon=0,1, b_{0}=0,5, q_{\mathrm{KB}}\left(0, b_{0}\right)=b_{0}$.

Величины $t_{C}$, отвечающие уравнению (9) при фиксированном $\sigma$, отображены в нижеследующей таблице и на рисунке.

\begin{tabular}{c||c|c|c|c|c|c|c}
\hline$\sigma$ & 0,01 & 0,02 & 0,05 & 0,06 & 0,07 & 0,08 & 0,09 \\
\hline$t_{C}(\sigma)$ & 230,752 & 149,85 & 81,3265 & 68,9434 & 54,2274 & 50,4592 & 50,2557 \\
\hline \hline$\sigma$ & 0,1 & 0,11 & 0,12 & 0,13 & 0,14 & 0,15 & \\
\hline$t_{C}(\sigma)$ & 50,1196 & 50,0159 & 49,9318 & 49,861 & 49,7999 & 49,746 & \\
\hline
\end{tabular}

При помощи методов регрессионного анализа определена зависимость $t_{C}(\sigma)$ (см. рисунок): $t_{C}=25,2086 / \sqrt{\sigma}-27,6926$, что соответствует оценке асимптотической зависимости, полученной выше: $t_{C}=O(1 / \sqrt{\sigma})$, при $\sigma \rightarrow 0$.

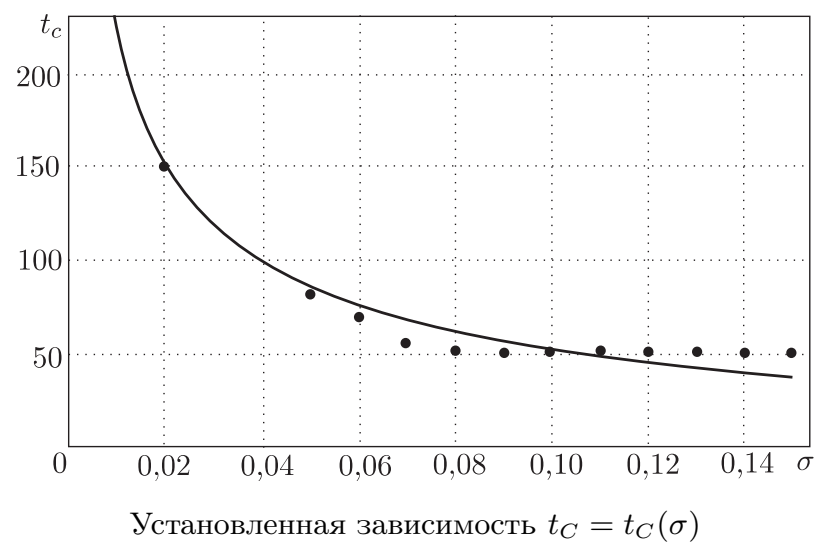

Результаты. В работе с помощью функционального подхода найдена усредненная траектория ангармонического осциллятора. Также проведено сравнение полученной траектории с классической, рассчитанной с помощью методов теории возмущений. В ходе сравнения найдена зависимость времени, за которое модуль разности двух траекторий впервые станет равным некоторому пороговому значению как функции удвоенной величины дисперсии начального значения координаты. 
Автор выражает глубокую признательность И.В. Воловичу за обсуждение работы и поддержку при её выполнении.

Работа выполнена при поддержке РФФИ (проекты №№ 08-01-00727-а, 09-01-12161офи-м), конкурса ведущих научных школ (проект НШ-3224.2008.1).

Автор также выражает благодарность за поддержку на конференции «Математическая физика и ее приложения - 2010» лабораторией математической физики СамГУ, грантами АВЦП 3341 и 10854 и контрактом ФЦП 2173.

\section{БИБЛИОГРАФИЧЕСКИЙ СПИСОК}

1. Волович И. В. Проблема необратимости и функциональная формулировка классической механики // Вестн. Сам. гос. ун-та. Естественнонаучн. сер., 2008. №8/1(67). C. 35-55, arXiv: 0907.2445 [cond-mat.stat-mech]. [Volovich I. V. Time Irreversibility Problem and Functional Formulation of Classical Mechanics // Vestn. Samar. Gos. Univ. Estestvennonauchn. Ser., 2008. no. 8/1(67). Pp. 35-55].

2. Volovich I. V. Randomness in Classical Mechanics and Quantum Mechanics // Found. Phys., 2010. Vol. 41, no. 3. Pp. 516-528.

3. Козлов В. В. Ансамбли Гиббса и неравновесная статистическая механика. М., Ижевск: Регулярная и хаотическая динамика, 2008. 204 с. [Kozlov V. V. Gibbs ensembles and non-equilibrium statistical mechanics. Moscow, Izhevsk: Regulyarnaya i Khaoticheskaya Dinamika, 2008. 204 pp.]

4. Volovich I. V. Functional mechanics and time irreversibility problem/ In: Quantum BioInformatics III. From Quantum Information to Bio-Informatics (Tokyo University of Science, Japan, 11-14 March 2009)/ QP-PQ: Quantum Probability and White Noise Analysis, 26, 2010. Pp. 393-404.

5. Trushechkin A.S., Volovich I. V. Functional classical mechanics and rational numbers // p-Adic Numbers, Ultrametric Analysis, and Applications, 2009. Vol.1, no.4. Pp. 361-367, arXiv: 0910.1502 [math-ph].

6. Трушечкин A.C. Необратимость и роль измерительного прибора в функциональной формулировке классической механики // TMФ, 2010. Т. 164, № 3. С. 435-440; англ. пер.: Trushechkin A.S. Irreversibility and the role of an instrument in the functional formulation of classical mechanics// Theoret. and Math. Phys., 2010. Vol.164, no. 3. Pp. 1198-1201.

7. Волович И. В. Уравнения Боголюбова и функциональная механика // TMФ, 2010. T. 164, №3. С. 354-362; англ. пер.: Volovich I. V. Bogoliubov equations and functional mechanics // Theoret. and Math. Phys., 2010. Vol. 164, no. 3. Pp. 1128-1135.

8. Inoue K., Ohya M., Volovich I. V. Semiclassical properties and chaos degree for the quantum baker's map // J. Math. Phys., 2002. Vol. 43, no. 2. Pp. 734-755.

9. Lichtenberg A. J., Lieberman M. A. Regular and Stochastic Motion / Applied Mathematical Sciences. Vol. 38. New York: Springer-Verlag, 1983. 449 pp.; русск. пер.: Лихтенберг А., Либерман М. Регулярная и стохастическая динамика. М.: Мир, 1984. 528 с.

10. Arnol'd V.I., Avez A. Problèmes ergodiques de la mécanique classique (in French)/ Monographies internationales de mathematiques modernes. Vol. 9. Paris: Gauthier-Villars, 1967. 243 рр.; русск. пер.: Арнолъд В.И., Авеи, А. Эргодические проблемы классической механики / Регулярная и хаотическая динамика. Т. 11. Ижевск: Регулярная и хаотическая динамика, 1999. 281 с.

11. Боголюбов Н.Н., Митропольский Ю.А. Асимптотические методы в теории нелинейных колебаний. М.: Гостехиздат, 1955. 447 с.; англ. пер.: Bogoliubov N. N., Mitropolsky Y. A. Asymptotic methods in the theory of non-linear oscillations. New York: Gordon and Breach, 1961. $537 \mathrm{pp}$.

12. Birkhoff G., MacLane S. A Survey of Modern Algebra. New York: Macmillan Publishing, 1977. $512 \mathrm{c}$.

Поступила в редакцию 21/XII/2010; в окончательном варианте - 27/II/2011. 


\section{MSC: 82C05}

\section{ON CLASSICAL AND FUNCTIONAL APPROACHS TO MECHANICS}

\section{E. V. Piskovskiy}

Moscow Institute of Physics and Technology,

9, Inststitutskiy per., Dolgoprudniy, Moskovskaya obl., 141700, Russia.

E-mail: piskovskiy@mi.ras.ru

In this paper the relevance of the classical trajectory of the anharmonic oscillator and the average trajectory obtained within the functional approach is considered. Dependence of threshold time of divergence of trajectories on dispersion of initial values is derived.

Key words: functional mechanics, classical mechanics, model system, anharmonic oscillator, threshold time.

Original article submitted 21/XII/2010; revision submitted $27 / \mathrm{II} / 2011$.

Evgeny V. Piskovskiy, Postgraduate Student, Faculty of Control and Applied Mathematics. 